\title{
Handover location accuracy for travel time estimation in GSM and UMTS
}

David Gundlegård and Johan M Karlsson

\section{Linköping University Post Print}

\section{Tweet}

N.B.: When citing this work, cite the original article.

Original Publication:

David Gundlegård and Johan M Karlsson, Handover location accuracy for travel time estimation in GSM and UMTS, 2009, IET Intelligent Transport Systems, (3), 1, 87-94.

http://dx.doi.org/10.1049/iet-its:20070067

Copyright: Institution of Engineering and Technology (IET)

http://www.theiet.org/

Postprint available at: Linköping University Electronic Press

http://urn.kb.se/resolve?urn=urn:nbn:se:liu:diva-16517 


\title{
Handover Location Accuracy for Travel Time Estimation in GSM and UMTS
}

\author{
David Gundlegård \\ Johan M Karlsson \\ Department of Science and Technology, \\ Linköping University, \\ SE-601 74 Norrköping, \\ Sweden
}

\begin{abstract}
In this paper, field measurements from the GSM and UMTS networks are analysed in a road traffic information context. The measurements indicate a potentially large improvement using UMTS signalling data compared to GSM regarding handover location accuracy. These improvements can be used to generate real-time traffic information with higher quality and extend the geographic usage area for cellular based travel time estimation systems. The results confirm previous reports indicating that he technology has a large potential in GSM and they also show that the potential might be even larger using UMTS. Assuming that non vehicle terminals can be filtered out, that vehicles are tracked to the correct route and that handovers can be predicted correctly, a conclusion from the experiments is that the handover location accuracy in both GSM and UMTS will be sufficient to estimate useful travel times, also in urban environments. If there is a scalable way of doing this filtering, tracking and prediction in urban environments is not clear to the authors today.
\end{abstract}




\section{Introduction}

The behaviour of the GSM network when it comes to generating road traffic information has been analysed for more than a decade, e.g in [1-3]. However, the UMTS network has not been a target for the same analysis, which means that it is not clear how the different characteristics of the UMTS network, compared to the GSM network, will affect the quality of the generated traffic information. Efforts to estimate traffic information from cellular networks started in mid 1990's, when the research project CAPITAL was initiated [4]. The projects following CAPITAL have taken many different approaches to extract information from the cellular networks, and several pilot projects are currently active in, among other countries, USA, Israel, Sweden and the Netherlands. A general travel time estimation algorithm can include the following basic steps: location data collection, identification of vehicle located terminals, map matching, possible route determination and travel time calculation.

In addition to these basic steps the algorithm should be able to compensate for anomalies like terminals in a bus with a separate lane, terminals on trains, terminals on motorcycles or bicycles etc. It is very difficult to filter out bicycles and motorcycles, which means that they will bias the travel time estimation. However, recently there have been efforts in trying to filter out public transports using e.g. time-tables [5]. With knowledge of the sections with separate lanes other ad-hoc filtering methods could most likely improve accuracy further. Map matching in this context is analysed in e.g. [6]. The route determination problem is discussed in e.g. [7, 8]. A survey and a detailed description about the differences in location data for generating road traffic information from the GSM and UMTS networks can be found in [9]. Recent extensive surveys of the area can be found in $[10,11,12,13]$.

Although the technology of using cellular networks to estimate road traffic information has been subject for analysis for quite some time, it is still far from being mature. It is not clear what to expect from these systems in terms of accuracy, availability and coverage. The potential of the system is although quite clear, it is possible to retrieve a lot of traffic data in a cost efficient way, i.e. by using existing signalling data without the need to invest in sensor infrastructure. Evolving communication systems will change communication patterns, and both the design of the communication system and the communication pattern of the users will affect the potential of estimating road traffic information from cellular networks. In order to realise a safer and more efficient transportation system, it is likely that vehicles need to communicate more frequently with each other and with some kind of traffic control centre. 
This traffic generates cellular network signalling data that can be very useful in order to estimate road traffic information in a cost efficient way. Interestingly, when vehicles communicate more, these systems will generate better traffic information. Better traffic information will probably generate more communication, which once again will generate better traffic information, and this can be seen as a positive spiral of traffic information collection.

This paper focus on comparing handover location information between GSM and UMTS networks in two different environments, a sparse urban environment with slow traffic and a suburban highway environment with higher speed limit. The handover location data can be an integral part when estimating road traffic information using passive monitoring of cellular systems. In order to estimate a travel time or average speed after the correct route has been identified, two well defined locations are needed. The time between these locations are then used as travel time for the segment or for calculation of average segment speed. The locations can be defined by any kind of event that can be detected through the cellular network and relates to a certain geographic position, e.g. a certain signal strength pattern or a handover. Hence, the handover location accuracy estimation can be used to assess the potential of both GSM and UMTS in estimating road traffic information. It can be seen as a lower bound on potential accuracy, since dedicated processing of raw data, i.e. measurement reports, might generate more accurate locations. On the other hand, due to the fact that handover locations are identified with a large number test drives, it can also be seen as an upper bound on handover location accuracy. Handover location accuracy should not be mixed up with travel time accuracy, since a number of factors except handover location accuracy affect the final travel time estimation, e.g. tracking the terminals to the correct route and filtering out non-vehicle terminals.

\section{Measurement objectives}

Numerous sen sor types are available to measure road traffic state information such as speed, density and flow. Stationary sensors, e.g. inductive loops and IR sensors, measure vehicle traffic parameters in a given location. Floating sensors are located in vehicles and measure the parameters for a given vehicle at different locations. The vehicles that are equipped with sensors are often referred to as probe vehicles or floating cars. License plate matching technologies measure the travel time between video camera locations. Different types of sensors have different advantages and drawbacks. Which sensor that should be used is dependent on traffic conditions, road network structure and financial aspects, but also the main application of data is relevant to this 
choice. Two types of applications can be distinguished, incident detection and travel time estimation.

The performance of different systems for traffic information depends on a number of factors related to the measurement procedure and the number of sensors, but also on how the performance metrics are defined. Ideally we would want to know our own travel time given that we start our travel at a certain time in the near future. However, that is a quite challenging task, and the aim is currently to report the historical travel time dating e.g. five minutes back. Travel times fluctuate due to individual driving patterns and it is not obvious if we want to know the lowest, average or highest travel time in the reporting interval. Speed fluctuations due to driver behaviour decreases when the road gets more congested and the incident or slowdown detection is another important application. A common performance metric is the time for a system to detect an incident. The definition of an incident varies, e.g. $40 \%$ slow down, and affects the performance. For stationary sensors the time to detect an incident depends on the time it takes for the incident to propagate to the sensor. In this case the sensor spacing is the crucial factor. For floating sensors the time to detect an incident depends on the time for the sensors to propagate to the incident. This time is highly correlated to the number of vehicles with sensors (penetration) and the reporting interval of the sensors. Using signalling data from cellular networks gives a potentially high number of floating sensors with a very short reporting interval. The issue in these kinds of systems is the relatively low location accuracy and the rest of this paper investigates this issue further. The best results are most likely obtained when fusing information from several sensors with different characteristics.

\section{Location data in GSM and UMTS}

The type of location data available for a terminal in GSM and UMTS depends on the state of the terminal. The state, on the other hand, depends on how the terminal is used, i.e. used for surfing the web, making telephone calls etc. In both GSM and UMTS, terminals used for real-time services generate the most detailed location data and we will focus on this kind of data throughout the paper.

Terminals used for real-time services continuously send measurement reports about the radio environment in order to assist the network in the handover decision. These measurement reports together with handover points can be used to track the route of the vehicle and calculate travel times. The measurement reports in GSM are sent every $480 \mathrm{~ms}$ and they contain the signal 
quality of the serving base station, signal strength of surrounding base stations and a timing advance (TA) value that gives a rough estimation of the distance to the serving base station. The measurement reports in UMTS are more flexible, the network can determine the reporting strategy dynamically. A large difference compared to GSM is the use of event triggered measurement reports, i.e. the mobile terminal makes measurements and sends measurement reports when an event has occurred. The event triggered reports can be replaced or combined with periodic reports.

In UMTS the periodic measurement report interval is configurable between 0.25 and 64 seconds, depending on radio environment and the state of the mobile terminal [12]. The frequency of event triggered reports are dependent of the frequency of actual events, e.g. a new radio link addition to the active set, but also on the operator configurable parameters time-to-trigger, hysteresis and and offset value. More detailed information on UMTS measurement reports can be found in e.g. $[14,15,16]$. Signal strength and quality of serving base station(s) are similar to the ones in GSM. The maximum number of surrounding base stations that can be measured is increased from 6 in GSM to 32 in UMTS. A TA value is not calculated in UMTS (WCDMA) networks since it is not a TDMA based system, but other time alignment measurements are available, e.g. round trip time and time difference between base stations [15].

An important difference between GSM and UMTS is the possibility to use soft handover in UMTS. This means that a terminal can be connected to several base stations simultaneously in UMTS, whereas in GSM the terminal is only connected to one base station at the time. To track a vehicle, both measurement reports containing radio parameters and handover points can be used. When it comes to calculating travel times it is very important to have two accurate estimations of the vehicle's position in order to make a good estimation of the travel time between those points. The handover points in GSM are a good candidate to estimate those positions. However, in UMTS the terminal will not change from one base station to another, instead radio links will be added to and removed from the terminals active set and the purpose of this paper is to verify how efficiently these soft handover points can be used to tie the vehicle to a certain position in the road network.

\section{Location experiments}

The aim of the location experiments is to compare the location accuracy of handover points in GSM and UMTS. The first experiments were carried out on a 900 meters long street segment in a "sparse" urban environment. The segment was driven fifteen times back and forth with test equipment for GSM 
and UMTS (Ericsson TEMS ${ }^{*}$ Investigation 7.0) and a GPS receiver. Signalling data was collected from a GSM terminal and a UMTS terminal simultaneously, both with ongoing telephone calls. For comparison another test run was made in a different environment. The second test was performed in a suburban highway environment with less complex GSM cellular structure. Since handovers are analysed, we assume a passive monitoring approach for travel time calculations, i.e. existing signalling traffic from the cellular networks are used to estimate travel times.

In the sparse urban environment eight handover zones were detected for UMTS (see Fig. 1). In six of the handover zones, a specific radio link was added, removed or both in every test run. In handover zone 7 , a soft handover occurred in twelve of the fifteen test runs and in handover zone 8 , a soft handover was completed in nine test runs. During the fifteen test runs the UMTS terminal was connected to four different base stations. The main changes between the different test runs were the number of times a radio link was added within a handover zone and whether a handover occurred at all in handover zone 7 and 8.

For the GSM test runs, four handover zones could be distinguished (see Fig. 2). During the fifteen test runs, the GSM terminal was connected to nine

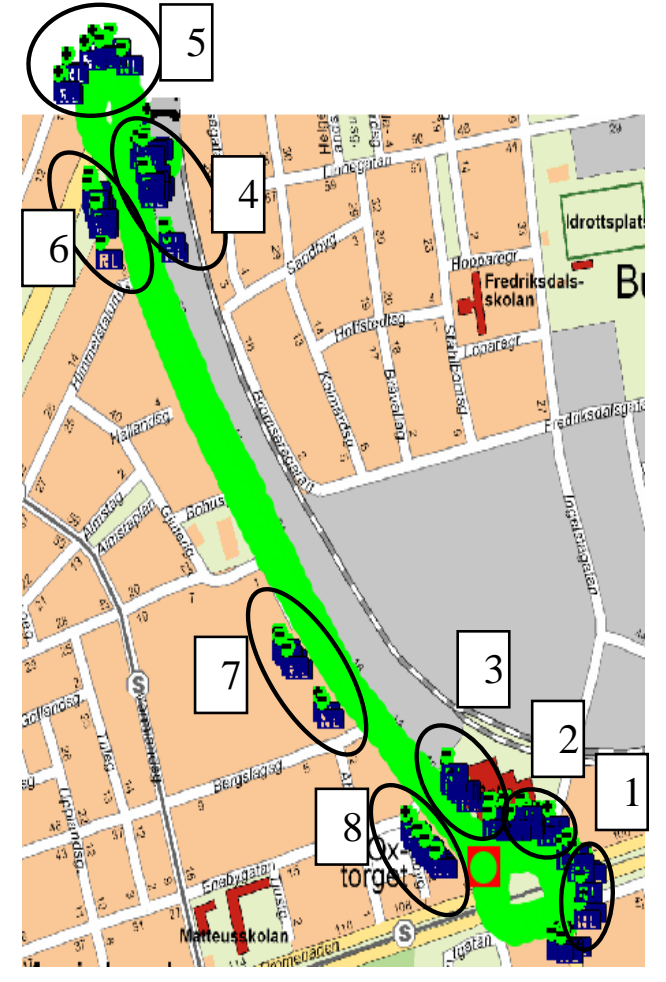

Figure 1. Handover zones in UMTS

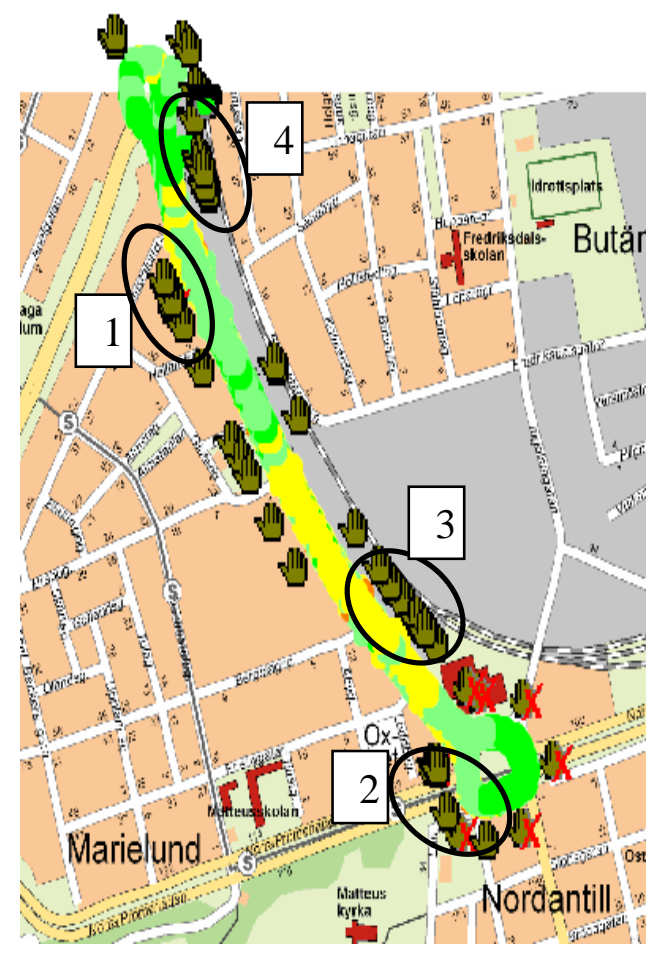

Figure 2. Handover zones in GSM.

${ }^{*}$ TEMS $=$ Telecommunication Management System 
different cells. In the four handover zones a handover occurred in every round, however, no specific handover, i.e. between the same two cells, occurred in every test run.

Figure 1 and 2 indicate quite large differences in the behaviour of the GSM and UMTS networks regarding handovers. In the suburban highway environment several UMTS and GSM handover zones were detected. Three of these handover zones for both GSM and UMTS were analysed in detail using seven test runs on a $1.5 \mathrm{~km}$ road segment. Next, a more detailed analysis is made regarding the differences in terms of handover location accuracy and how it can affect the estimation of travel times.

\section{Handover location accuracy}

Several approaches are possible when determining the location of a handover in UMTS. Since soft handover is used, radio links are added, removed or replaced in the active set. The typical scenario in the experiments was that a radio link was added and removed several times in a handover zone. Normally the newly added radio link becomes stronger somewhere within the handover zone and is eventually the only one remaining, but this is not necessarily the case. Straightforward methods to determine the handover location are to use the first radio link addition or removal, the last radio link add ition or removal or the point when a new radio link is the strongest. Using the radio link additions possibly generates more handover points, but the accuracy might be better using the point in time when a new radio link is stronger. In practice, implementation details will settle which data or combination of data that will be used. An insight of the experiments is that using all radio link additions or points where a new radio link is stronger renders a lot of ambiguity within handover zones, and therefore the first or last radio link addition in a handover zone were used in the location accuracy calculations below.

In GSM hard handovers are used, which means that there is no doubt about where a handover is located. Of course, several stages of the handover could be defined as the actual handover point, but that is of less importance as long as the same definition is used for both calibration and estimation.

\section{Sparse Urban Environment}

The percentage of times a specific handover is completed in the same handover zone, driving the same route several times, is in this paper referred to as consistency. The consistency of handover points will affect the travel time estimations. If a predicted handover does not occur, it will feed the system 
with no data if the handover sequence cannot be identified or corrupt data if the vehicle is tracked to the wrong route. The consistency of the different handover points for GSM and UMTS are shown in Figure 3.

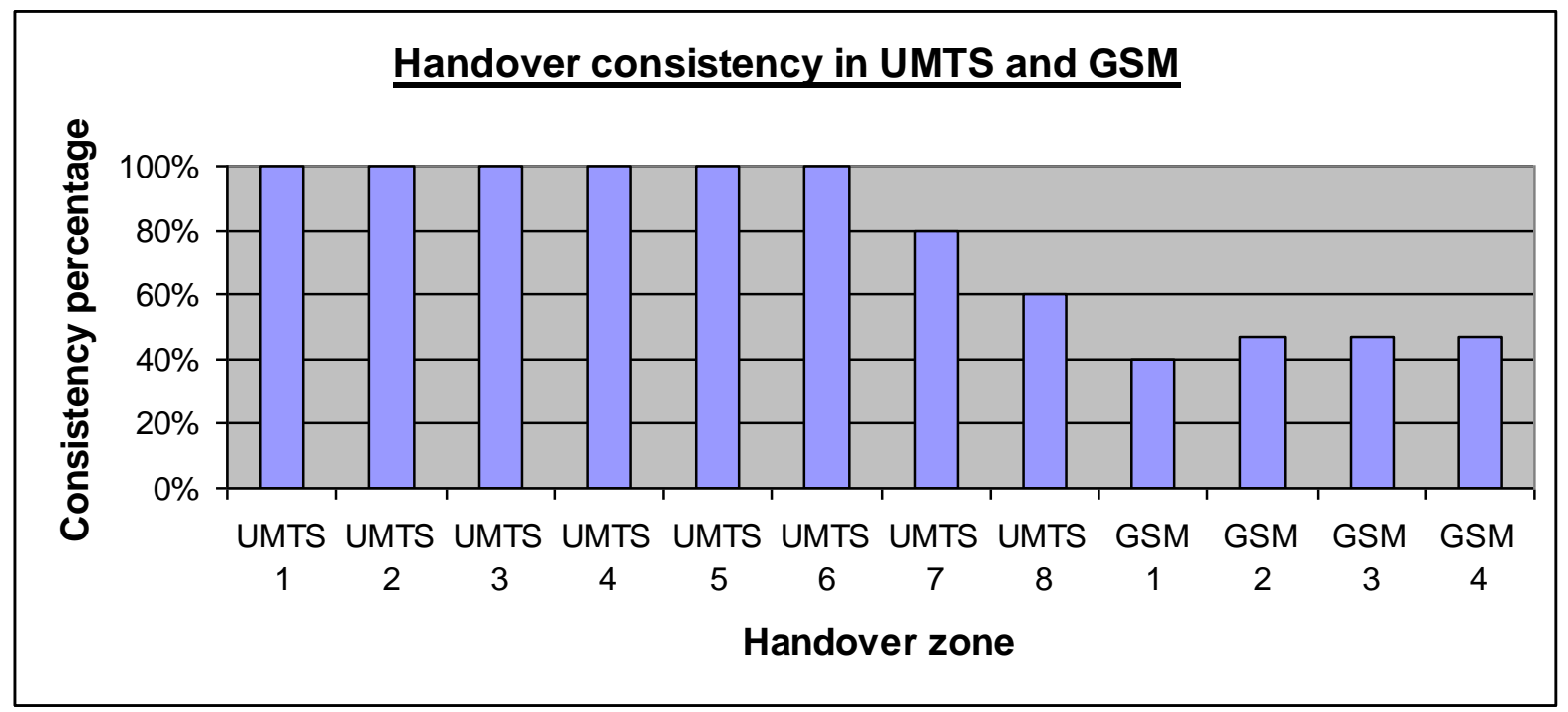

Figure 3. Sparse urban environment. Handover consistency in UMTS and GSM.

Compared to the UMTS case, the GSM handovers were much more scattered. Several handovers occurred outside the handover zones and the handovers within the zones were between different cells. The reason that two of the UMTS zones did not have $100 \%$ consistency was that no soft handover occurred in these zones in several of the test runs. The GSM inconsistency on the other hand, was due to handover between different cells in the same handover point. A reason for this can be that the UMTS network has more free capacity than the GSM network, and there is a possibility that the inconsistency of the GSM handovers are due to cell capacity limitations. However, the test runs were driven at two different days and times and it is not likely that the network was congested in both occasions. Since the results were sim ilar for GSM in both test runs, a congested network does not seem to be a good explanation. A more probable explanation might be the different cell structures of GSM and UMTS in the test area. Due to relatively few subscribers in the UMTS network, a hierarchical cell structure has not yet been developed, as often is the case for the GSM network. A hierarchical cell structure might explain some of the scattered handovers and the fact that several different handovers were detected in the same handover zone. 
The handover location accuracy is assessed using data from the 15 test runs to calculate an average handover point and measure the deviation from this point in the different test runs. The deviation is measured from the average point of all handovers within a handover zone. It should be noted that since the GSM handovers were very inconsistent, handovers between different cells will be grouped to the same average point, which will affect the GSM handover location accuracy measurements negatively. The handovers in UMTS are defined as the first or last radio link addition depending on handover zone. Missing handovers in a zone are ignored in the accuracy measurements, i.e. the consistency and accuracy diagrams should be assessed and analysed independently. All handover positions are mapped to the onedimensional space of the traversed road segment. The positioning error of the GPS receiver is included in all measurements.

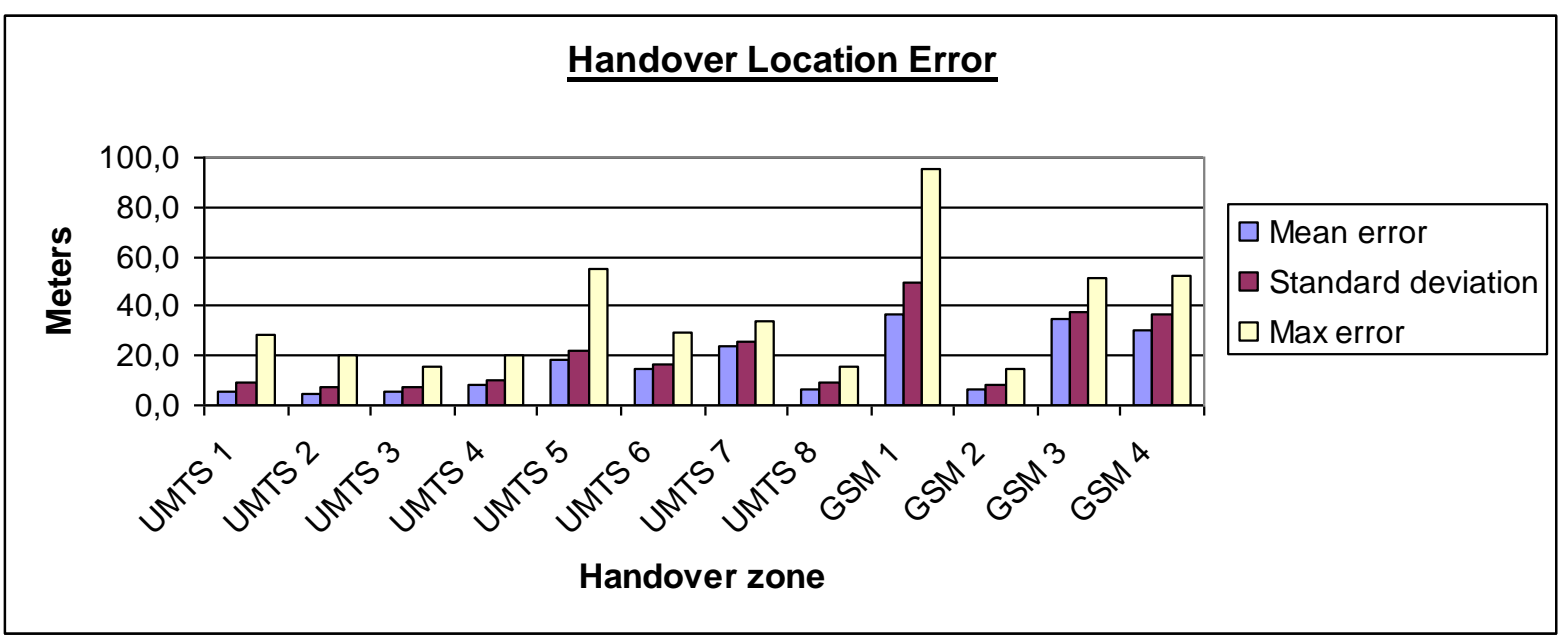

Figure 4. Sparse urban environment. Mean and max handover location error (in deviation from average handover point) together with the standard deviation of the measurements.

Figure 4 shows that the accuracy is quite good for a majority of the handover zones in both GSM and UMTS. The mean error is below 20 meters for more or less all of the UMTS handover zones, and below 40 meters for the GSM zones. However, a large variation in accuracy between different handover zones can also be seen, as well as a large difference between mean and maximum error. The large maximum error of handover zone 5 in UMTS was due to an ou tlier in one of the rounds. The UMTS accuracy seems in general much better than for GSM, which, as mentioned previously, partly could be explained by different cell structure and traffic pattern in the area. It should be noted that it is possible that the soft handover technique used in UMTS might render much 
more stable handover points compared to GSM, but it is not clear from the experiments what will happen if the traffic pattern and cell structure of the UMTS network changes. In the measurements the true value of the average handover point for the data set is used to calculate the location error. This value will not be known in a travel time estimation system, but instead estimated using test runs or coverage maps.

\section{Suburban Highway Environment}

The previous hypothesis that the low accuracy and consistency of the GSM handovers is due to a complex hierarchical cell structure is evaluated with a second test in a different road and radio environment. This environment has most likely a flat GSM cell structure and the road is a highway with speed limit $90 \mathrm{~km} / \mathrm{h}$. In this environment it is also possible to evaluate how the vehicle speed affects the handover accuracy. It is possible that a higher speed gives a larger handover zone due to larger movement between measurement reports. The results of the test runs are shown in Figure 5 below.

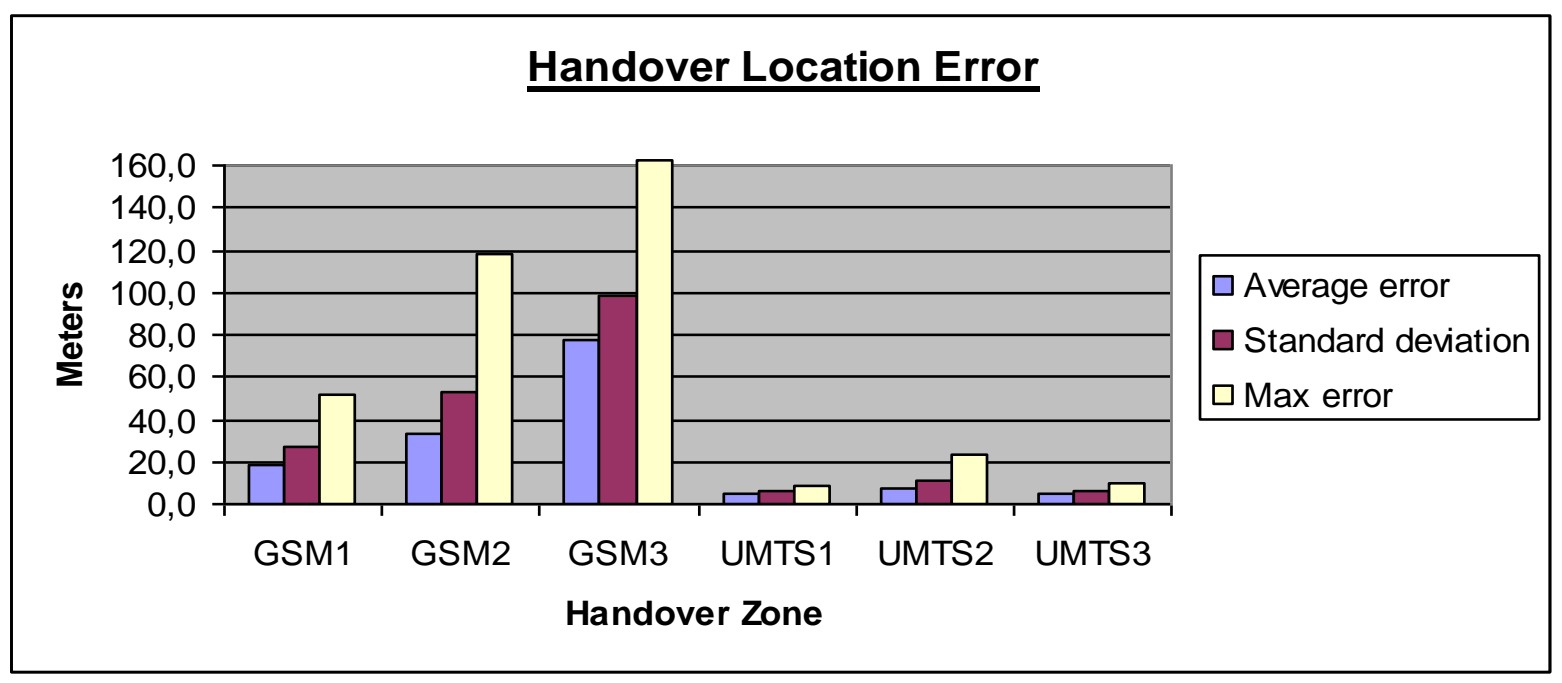

Figure 5. Suburban highway environment. Mean and max handover location error (in deviation from average handover point) together with the standard deviation of the measurements.

The consistency for all evaluated handover zones is $100 \%$ for both GSM and UMTS. This implies that the low consistency for GSM in the urban environment can be due to the cell structure. The location accuracy of the UMTS handovers is in the same order as the best ones from the urban environment, i.e. very good. On the other hand, the GSM measurements have a large variation where one handover zone has an average error of 20 meters where as another one has an average error of almost 80 meters. From the results we can 
expect that the vehicle speed does not have a dramatic effect on the accuracy, the handover consistency can be very affected by cell structure and the UMTS handovers seem to have much better location accuracy.

Comparing the location error of all 68 handovers performed in GSM and all the 99 handovers performed in UMTS, it is possible to draw better conclusions regarding a general difference in handover location accuracy. The average location error of all handovers in GSM is $23.1 \mathrm{~m}$ and $6.3 \mathrm{~m}$ in UMTS. Considering the null hypothesis that there is no difference in the average hand over location error value between GSM and UMTS, it can be discarded with a two-sided p-value of less than $10^{-6}$. This means that it is very likely that the long term average value of handover location error is lower in UMTS compared to GSM, considering the same network conditions. It is also quite likely that this statement is valid for other network conditions.

\section{Potential Travel Time Accuracy}

The purpose of calculating the handover location error, as described above, is to evaluate the potential of a travel time estimation system based on monitoring active cell phones. By using two handover locations, a travel time can be calculated for a vehicle passing between them. The accuracy and the consistency of the handover location will be a very important factor for the travel time accuracy. It is important to mention that the travel times are calculated under the assumption that all vehicles are tracked to the correct road segment and that it is identified as a vehicle, hence the term potential travel time accuracy is used. In a real system, these tasks are typically very challenging, especially in an urban environment. This problem is discussed in detail in e.g. [6, 7].

Figure 6 shows an example of estimated and actual handover locations during a test run. $D_{E}$ is the distance between the estimated handover locations. These are typically calibrated using coverage maps or test runs. In this case $D_{E}$ is the distance between the average handover locations, since they were estimated based on all of the test runs. $D_{i}$ is the distance between the actual handover points during test run $\mathrm{i}$, where $i \in 1 . .15$. The actual travel time during test run $i\left(\mathrm{~T}_{\mathrm{i}}\right)$ is simply the time to travel the distance $\mathrm{D}_{\mathrm{E}}$, whereas the estimated travel time during test run $i\left(\mathrm{~T}_{\mathrm{Ei}}\right)$ is the time to travel the distance $\mathrm{D}_{\mathrm{i}}$. 


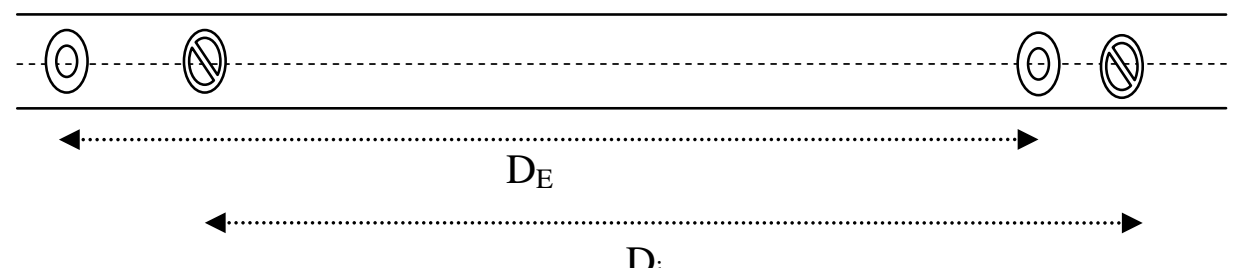

$\mathrm{D}_{\mathrm{i}}$

Estimated location
of handover

Figure 6. Overview of estimated and actual handover locations for travel time estimations.

Figure 7 and 8 shows the actual travel time $\left(\mathrm{T}_{\mathrm{i}}\right)$ and the estimated travel time $\left(\mathrm{T}_{\mathrm{Ei}}\right)$ together with actual mean speed and estimated mean speed for a street segment traversed in the test runs. The actual and estimated travel times and mean speeds are calculated using the TEMS logfile between handover zones 3 and 4 in both the GSM and the UMTS network.

In test run two for GSM no estimation is shown since the handover was blocked. In fact, also handover blocking events could be u sed as location data, but using them as input to travel time estimation can be risky due to increased possibility of extreme outliers. Also test run two in UMTS lacks travel time estimation, in this case the reason was equipment disconnection just before handover zone 3 .

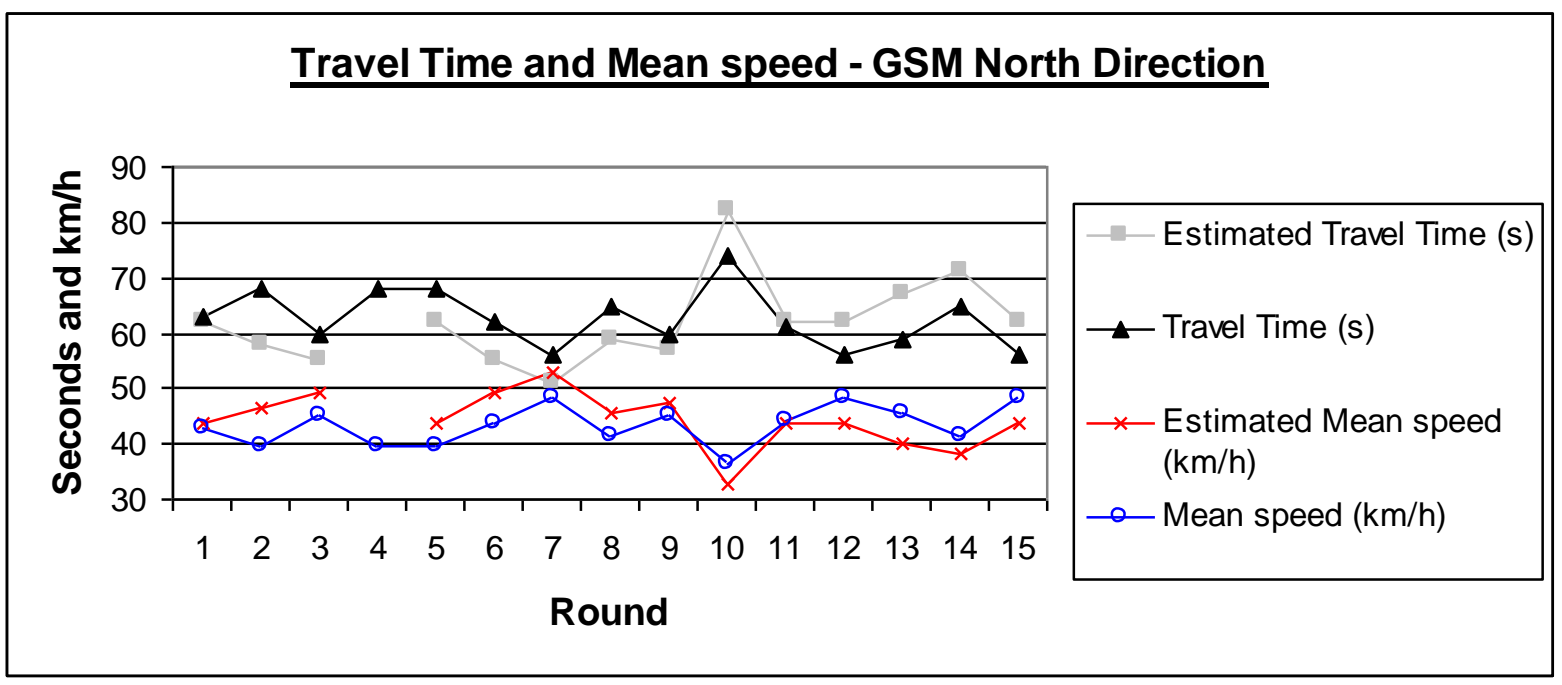

Figure 7. Travel times and mean speed estimated with GSM hand over zones 3 and 4. 
Travel Time and Mean speed - UMTS North Direction

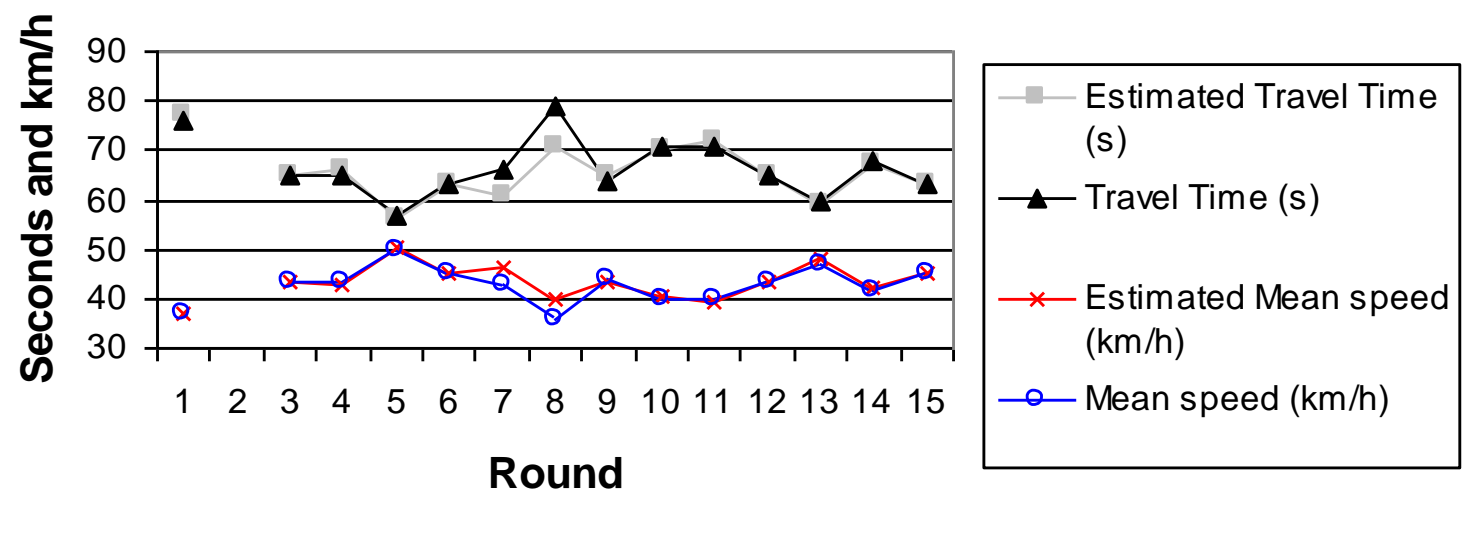

Figure 8. Travel times and mean speed estimated with UMTS handover zones 3 and 4.

Interestingly there seems to be a correlation between the first and second handover location, i.e. when the handover to a certain cell is late, the handover from this cell to the next cell is also late. If there is such a correlation, the travel time error will be smaller than indicated by the handover location error, since the travel time error is defined as the absolute difference $\left|T_{i}-T_{E i}\right|$.

As can be seen in Figure 6 above, the estimated UMTS travel times follow the actual travel times extremely well. However, as can be seen in Figure 5, also the estimated GSM travel times follow the actual travel times in a good way. Four main factors affecting travel time accuracy are handover location accuracy, handover consistency, number of available probes in a time interval and road segment length. Handover location accuracy and consistency are discussed above, but also the number of available probes and the road segment length are important for travel time accuracy, interestingly they are also correlated. Increasing the road segment length will make the handover location error less significant and yield better travel time accuracy. More vehicle probes will give a better accuracy due to the possibility of averaging the travel time value between several probes in a travel time reporting interval. However, increasing the road segment length will also decrease the number of ongoing telephone calls or data sessions that completes the whole segment, which gives less probes for averaging. Shorter road segments for travel times also lead to more detailed traffic information, which might be useful in for example urban environments.

The road segment lengths (distance between average handover points) of the experiments were 750 and 785 meters for GSM and UMTS, respectively. 
The experiments indicate that it is possible to make good travel time estimations from cellular networks for this segment length, even shorter segment lengths are possible depending on radio environment, number of probes and accuracy requirements. Another factor that will affect the travel time accuracy is the average vehicle speed in the handover zones. If a vehicle is travelling slowly in a handover zone, a small location error can affect the travel time quite drastically. This problem will typically be common when deploying this kind of systems in urban environments.

\section{UMTS Characteristics and Potential Benefit}

In the coming years there will be many countries with a combination of UMTS and GSM terminals. Most likely the share of UMTS terminals will grow. In Sweden, for example, the number of new UMTS terminals has passed the number of new GSM terminals. Hence, using also UMTS terminals for travel time estimation will increase the number of floating sensors in the system, which is an important factor when it comes to both travel time accuracy and time to incident detection. Increasing the number of vehicle samples in a travel time reporting interval is very important for accuracy, especially since the system produces relatively noisy measurements.

The higher data rate and shorter delay together with dynamic measurement reporting in UMTS makes the network to react much faster to changes in the radio environment, which affects the location accuracy of network events. This, in combination with the soft handover principle that makes a radio link addition or removal without large delay, might be the reason to the much better UMTS location accuracy in the evaluated tests. Generally speaking there is also a better synchronization between base stations and mobile terminals in UMTS and (eventually) a more dense radio network which gives a potentially higher location resolution. This is independent of whether handovers or something else is used to determine specific locations of the mobile terminal on the road.

The higher location accuracy in the UMTS network can be used to make the travel time accuracy better or maintaining the relative accuracy while making the travel time segments shorter. Shorter travel time segments are necessary in e.g. urban environments and are also useful when detecting incidents.

The field tests show that the potential accuracy of the UMTS handovers is much better than for GSM. However, it should also be noted that there is much more information available in UMTS, leading to potential ambiguity and real-time data processing problems. As an example there were 243 radio 
link additions in UMTS in the seven test runs of the highway environment whereas the same test runs generated only 28 handovers in GSM.

\section{Conclusions}

Since the behaviour of the cellular networks is implementation and environmental dependent, it is difficult to make general conclusions from limited tests. However, valuable insights were made about the behaviour of the different networks when it comes to estimating travel times based on handover signalling data.

The experiments indicate relatively good handover location accuracy for both GSM and UMTS. The handover location accuracy and consistency was much better in UMTS than in GSM. A complex cell structure might be an explanation to the inconsistency in the GSM handovers, this can also explain some of the problems with system deployment in urban environments. More calibration work is therefore needed in the parts of the network that has a complex cell structure in order to predict handovers correctly. These parts of the network can easily be identified using the operator's cell plan. Assuming that non vehicle terminals can be filtered out, vehicles are tracked to the correct route and that handovers can be predicted correctly, a conclusion from the experiments is that the handover location accuracy will be sufficient to estimate useful travel times, also in urban environments. If there is a scalable way of doing this prediction and filtering in urban environments is not clear to the authors today.

As soon as there are enough probes, using GSM signalling data to estimate road traffic information on highways seems quite well established and promising. To do the same thing in urban environments is a more challenging task. Complementing GSM systems with UMTS signalling data will improve travel time estimation accuracy, and has the potential to extend coverage for the systems also to urban areas. After all, there is a lot more signalling data from vehicles available in urban environments, and it would be a waste not to use it.

Handover algorithms are not designed to make handover decisions in specific geographic positions, and are hence not optimised to determine a location. This implies that separate analysis of measurement reports can increase the accuracy of location events. However, this is traded against more data processing and the accuracy of handover locations can in this case give good input about the potential of the technology. 


\section{References}

[1] Bolla, R., and Davoli, F.: "Road Traffic Estimation from Location Tracking Data in the Mobile Cellular Network". Proceedings of WCNC, Chicago, September 2000, pp. 1107-1112

[2] Ygnace, J-L and Drane, C, "Cellular Telecommunication and Transportation Convergence," Proceedings of ITSC, Oakland, August 2001, pp. 16-22

[3] Karhumäki, T, “The Utilisation of GSM-Network in Travel Time Monitoring," ITS Workshop on Road Monitoring, Imperia, 2002

[4] University of Maryland Transportation Studies Center. Final Evalu ation Report for the CAPITAL-ITS Operational Test and Demonstration Program. University of Maryland, College Park, 1997

[5] Schollmeyer, R, Wiltschko, T, "Classification of Public Transport Vehicles using Cellular Mobile Radio Data, Proceedings of ITS Europe, Aalborg, 2007

[6] Hellinga, B, Liping, F, Takada, H, “Obtaining Traveller Information via Mobile Phone Location Referencing - Challenges and Opportunities, Proceedings of the Transportation Factor, 2003

[7] Mangold, S, Kyriazakos, S, “Applying Pattern Recognition Techniques based on Hidden Markov Models for Vehicular Positioning Location in Cellular Netw orks", Proceed ings of VTC '99, Amsterdam, 1999

[8] Hellebrandt, M, Mathar, R, "Location Tracking of Mobiles in Cellular Radio Networks", IEEE Transactions on Vehicular Technology, Vol. 48, No. 5, September 1999, pp. 1558-1562

[9] Gundlegård, D and Karlsson, J M, "Generating Road Traffic Information from cellular Networks - New Possibilities in UMTS", Proceedings of ITS-T, Chengdu, 2006

[10] Hellinga, B, Izadpanah, P, "An Opportunity Assessment of Wireless Moitoring of Network-Wide Road Traffic Conditions," Final report prepared for Ministry of Transportation of Ontario, 2007

[11] Subbarao, W.V. et. al, "Travel Time Estimation using Cell Phones for Highways and Roadways", Final report prepared for Department of Transportation, 2007

[12] Virginia Transportation Research Council, "Probe-based Traffic Monitoring State-of-the-Practice Report," NCHRP 70-01, 2005

[13] Qiu, Z, Cheng, P, "State of the Art and Practice: Cellular Probe Technology Applied in Advanced Traveler Information System, Proceedings of TRB, Washington, 2007 
[14] Hiltunen, K, Binucci, N, Bergström, J, “Comparison Between the Periodic and Event-Triggered Intra-Frequency Handover Measurement Reporting in WCDMA", Proceed ings of WCNC, 2000

[15] 3GPP, "Requirements for support of radio resource management", TS 25.133, v. 7.2.0, December 2005

[16] 3GPP, "Radio Resource Control (RRC); Protocol Specification" TS 25.331, v. 8.0.0, September 2007 